\title{
DETERMINANT REPRESENTATIONS OF APPELL POLYNOMIAL SEQUENCES
}

\author{
YONGZHI YANG
}

Abstract. In this paper, we develop a new representation for Appell polynomial sequence via determinants. In addition, we demonstrate a direct application of this determinant formula (representation) for Appell sequence by deriving a determinant formula for a power series of a function $f(t)$ in terms of the power series of its algebraic inverse function $1 / f(t)$.

Mathematics subject classification (2000): 15A15, 05A40, 11B68.

Keywords and phrases: Appell polynomial, determinant formula, Leibinz matrix.

\section{REFERENCES}

[1] A. Di Bucchianico, D. LoEB, A selected survey of umbral calculus, Electronic J. Combinatorics, 3 (2000), 1-34.

[2] D. Kalman, A. Ungar, Combinatorial and functional identities in one-parameter matrices, The American Mathematical Monthly 94 (1987), No. 1, 21-35.

[3] D. H. LEHMER, A new approach to Bernoulli polynomials, The American Mathematical Monthly 95 (1988), No. 10, 905-911.

[4] S. Roman, The Umbral calculus, Academic Press Inc., Orlando, Florida, 1984. 